\title{
Maxillary Sinusitis of Dental Origin
}

\author{
Ankit Patel and Anastasia Rachmanidou \\ ENT Department, University Hospital Lewisham, London SE13 6LH,UK
}

\begin{abstract}
We report a 53 year-old male who suffered 18 months of foul-smelling green nasal discharge, halitosis and a heavy discomfort behind his right eye. The symptoms were consistent with chronic rhinosinusitis. The patient had a right upper molar dental extraction shortly before the onset of symptoms. A radio-opaque foreign body was noted in his right maxillary sinus on CT. During surgery a tooth resembling foreign body was removed. The patient's symptoms completely resolved within weeks of the removal.
\end{abstract}

Key words: Maxillary sinusitis, foreign bodies, tooth, dental implants.

\section{Introduction}

The clinical relevance of this article is to remind healthcare professionals about the possibility of dental fragment migration and the sequelae which can result.

Objective Statement-The reader should understand the clinical implications of dental fragment migration into the sinuses.

The theoretical presence of dental fragments in the maxillary sinuses is well-known, along with the possibility of chronic sinusitis development. The majority of reported cases highlight symptomless patients where the foreign body is removed to prevent potential symptoms. We present a patient who suffered with debilitating symptoms for 18 months as a result of a dental fragment which hadn't been identified. When the causative factor was identified the problem was quickly resolved. This case aims to remind healthcare professionals to consider this cause when patients attend with refractory chronic sinusitis of acute-onset.

\section{Case Report}

A 53 year-old male was seen in the ENT outpatient clinic with an 18-month history foul-smelling green nasal discharge, halitosis and a pressure pain behind his right eye. The symptoms were exacerbated by leaning forwards or backwards and worse during the night. The

Correspondingauthor: Ankit Patel, Dr., MBBS, BSc (Hons), research field: ENT. E-mail: ankitpatel@doctors.org.uk. symptoms had been refractory despite multiple antibiotics. He denied any red-flag symptoms. The symptoms had started following a right tooth extraction. On examination he had a deviated nasal septum with evidence of chronic rhinitis. Examination was otherwise unremarkable.

A coronal paranasal CT scan (Fig. 1) was performed. It confirmed extensive sinusitis in the right maxillary antrum and some right ethmoid sinus mucosa thickening. It showed a radio-opaque focus in the maxillary sinus. A deviated septum was also noted.

The patient was booked semi-electively into theatres for functional endoscopic sinus surgery. There was evidence of chronic rhinosinusitis with purulent discharge from the right middle meatus and inflamed mucosa. A calcified lesion resembling a tooth was extracted from the right maxillary sinus.

The patient was seen routinely after 3-months in the outpatient clinic. His nasal discharge, halitosis and facial pressure had regressed. On examination there was no evidence of rhinitis. The patient was very pleased with the outcome.

\section{Discussion}

Chronic rhinosinusitis refers to inflammation of the nose and the associated paranasal sinuses which last for greater than 12 weeks. It is diagnosed subjectively on the basis of two or more of the following symptoms; nasal congestion, facial pain or pressure, nasal discharge, 


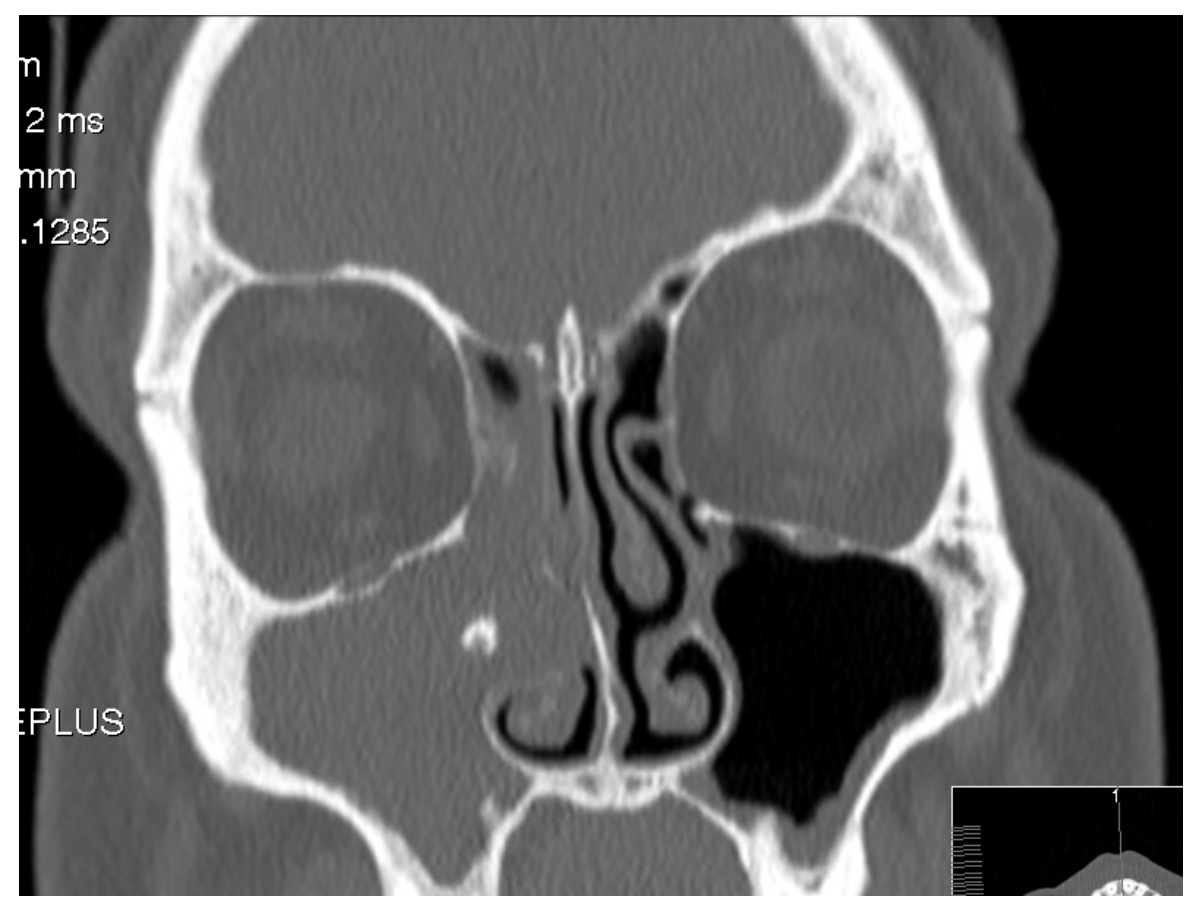

Fig. 1 Paranasal CT scan-Coronal view-Showing chronic right maxillary rhinosinusitis and a calcific focus.

and a reduced sense of smell. Patients should also have objective evidence of disease, either directly with an endoscope or CT-imaging - this identifies nasal polyp presence [1].

The aetiology and pathogenesis are not completely clear. Traditionally, it was believed to be an extension of inadequately treated acute sinusitis [5]. Anatomic variants, such as septal deviation have been suggested to play a role in the predisposition of some chronic rhinosinusitis cases [2]. Other exacerbating factors which have been advocated to play a role include air pollution, indoor dampness, and mould exposure, active and passive cigarette smoking [1]. Patients who suffer from allergic rhinitis (IgE mediated) have an increased risk of developing chronic rhinosinusitis on the basis that they have a greater sensitivity to perennial allergens [3].

The overall prevalence of chronic rhinosinusitis is estimated to be $10.9 \%$ [4]. Approximately $5 \%$ of chronic rhinosinusitis is the result of foreign bodies: $3 \%$ are of dental origin [6]. Given the close proximity between the maxillary sinus and maxillary teeth it is of little surprise that dental fragments can migrate into the sinus. They carry with them oral bacteria resulting in odontogenic sinusitis. Foreign bodies causing chronic rhinosinusitis most often result in unilateral symptoms.

The management of choice generally involves confirmation of a foreign body in the maxillary sinus by CT-scan, followed by functional endoscopic sinus surgery (FESS). FESS allows examination under anaesthesia and appropriate removal of the foreign body with minimal surgical trauma, resulting in reduced morbidity and complications [7].

Dental implant or fragment displacement into the maxillary sinus has been reported occasionally in the literature [8]. Most case reports are of dental implant migration with no signs or symptoms of infection. In all the cases the implant was removed due to the potential of sinusitis development. Other even rarer complications include pansinusitis, panopthalmitis and orbital cellulitis. It is for these reasons that foreign bodies identified in the sinuses should be removed as soon as possible. This requires a surgical intervention. In the majority the intranasal approach using FESS is the most appropriate option. Depending on the individual case an alternative is the intraoral approach 
where an opening is created in the anterolateral wall of the maxillary sinus [8]. It is known as the Caldwell-Luc procedure (performed by oral surgeons), however it is associated with significant morbidity. Both techniques aim to remove the dental foreign body and treat associated infections.

Chronic rhinosinusitis attributed to a dental fragment is a rare but well-known occurrence. The majority of patients identified with intrasinusal dental foreign bodies are asymptomatic. Symptomatic patients present with unilateral symptoms of chronic rhinosinusitis. They always require surgical management in order to prevent further sinus disease. The majority of cases can be appropriately managed using FESS.

\section{Summary}

- Chronic rhinosinusitis attributed to a dental fragment is a rare occurrence

- The majority of patients are asymptomatic

- Unilateral symptoms of chronic rhinosinusitis are often attributed to foreign bodies

- They always require surgical management to prevent sinusitis and further complications

The author(s) declare that thereis no conflict of interests regarding the publication of thisarticle.

\section{References}

[1] Hamilos, D. L. 2011.“Chronic Rhinosinusitis Epidemiology and Medical Management."American Academy of Allergy, Asthma \& Immunology. Doi10.1016/j.jaci.2011.08.004.

[2] Jones, N. S., Strobl, A., and Holland, I. 1997. "A Study of the CT Findings in 100 Patients with Rhinosinusitis and 100 Controls."Clin. Otolaryngol Allied Sci.22:47-51.

[3] Berrettini, S., Carabelli, A., and Sellari-Franceschini, S. 1999, et al. "Perennial Allergic Rhinitis and Chronic Sinusitis Correlation with RhinologicRisk Factors." Allergy54:242-8.

[4] Hastan, D., Fokkens, W. J., and Ba chert, C., et al. 2011."Chronic Rhinosinusitis in Europe-an Underestimated Disease."Allergy66: 1216-23.

[5] Piromchai, P., Kasemsiri, P., and Laohasiriwong, S. 2013, et al. "Chronic Rhinosinusitis and Emerging Treatment Options."International Journal of General Medicine6:453-64.

[6] Thevoz, F., Arza, A., and Jaques, B. 2000."Dental Foreign Body Sinusitis."Schweiz Med.Wochenschr 125:30-4.

[7] Tingsgaard, P. K., and Larsen, P. L. 1997.“Chronic Unilateral Maxillary Sinusitis Caused by Foreign Bodes in the Maxillary Sinus."UgeskrLaeger 159:4402-4.

[8] Fusari, P., Doto, M., and Chiapasco, M. 2013. "Removal of a Dental Implant into the Maxillary Sinus by Means of the Bone Lid Technique." Case Reports in Dentistry260707. Doi: 10.1155/2013/260707. 